\title{
MONITORAMENTO RESIDENCIAL UTILIZANDO O ZABBIX E O PADRÃO IEEE 802.15.4
}

\author{
W. ROMEIRO* E F. COSTA \\ Instituto Federal de Ciências e Tecnologias do Rio Grande do Norte \\ wr.romeiro@gmail.com*
}

Artigo submetido em setembro/2014 e aceito em fevereiro/2016

DOI: $10.15628 /$ holos.2016.2439

\section{RESUMO}

O objetivo deste artigo é relatar os aspectos do desenvolvimento do sistema de automação residencial que utiliza a comunicação sem fio por meio da tecnologia ZigBee para acesso aos pontos de automação, Podendo ser gerenciado a partir do protocolo de gerenciamento SNMP. Sistema esse que permite ao usuário obter uma maior distância entre os pontos de automação, como também, a integração com sistemas já existentes de monitoramento que utilizem o protocolo SNMP, sendo assim, podendo coletar e manipular informações desses pontos.

PALAVRAS-CHAVE: Monitoramento Residencial, ZigBee, Zabbix, Arduino.

\section{RESIDENTIAL MONITORING USING ZABBIX AND IEEE 802.15.4 STANDARD}

\begin{abstract}
The purpose of this article is to describe aspects of the home automation system development that uses wireless communication via ZigBee technology for access to automation points, It can be managed from SNMP
\end{abstract} obtain a greater distance between the automation points, as well as, integration with existing systems monitoring using SNMP, therefore, can collect and manipulate information from these points.

KEYWORDS: Residential Monitoring, ZigBee, Zabbix, Arduino 


\section{INTRODUÇÃO}

A automação residencial possui como premissa possibilitar a automatização de atividades habituais dos moradores de uma casa, com o objetivo de gerar um conforto para tais moradores. Essas atividades vão desde o acionamento de luminárias, acionamento automático de condicionadores de ar e de sistemas de segurança.

Hoje existem soluções proprietárias para sistemas de automação residencial, bem como soluções desenvolvidas por meio da adaptação de hardwares e softwares livres. Dessa forma o usuário é quem possui autonomia para escolher a melhor maneira de implementação.

A dificuldade hoje encontrada para a implantação de um sistema de automação depende da forma como será implantado o sistema, se esta será de forma centralizada ou distribuída, na forma centralizada a maior dificuldade é a modificação do sistema elétrico, enquanto na distribuída a dificuldade encontrada é na manutenção, pois vários dispositivos precisam ser configurados.

Neste artigo será abordado um sistema de automação residencial que utiliza da estrutura de gerenciamento do protocolo SNMP (Simple Network Management Protocol), que por sua vez permite recuperar essas informações dos pontos aplicados na automação residencial, sejam eles luminárias ou tomada elétricas, podendo assim a partir de um software de gerenciamento monitorar e mudar essas informações. Partindo da ideia do uso de hardware e software livre.

\subsection{Motivação}

Nos dias atuais, as pessoas estão cada vez mais ávidas de conforto e praticidade na realização de tarefas diárias, isso porque elas estão com menos tempo para executar tais tarefas, estão correndo com trabalho ou outros compromissos. Com isso, surge a domótica, que serve para dar conforto na realização das tarefas dentro de uma residência. Então tornar esse processo mais prático é o que evidencia a automação residencial, quando se fala no uso de tecnologias sem fio, a domótica ganha mais ênfase, visto que, durante a instalação ou manutenção não é necessária fazer grandes mudanças para implantar a automação residencial. Vale também salientar a facilidade de realizar tarefas mesmo não estando presente, como por exemplo, acender uma luz da casa, quando se estar viajando, para dar a sensação de que tem alguém presente na casa.

Dessa forma, a domótica é constituída por equipamentos que executam tarefas préprogramadas e assim proporcionam conforto e bem estar aos moradores desse ambiente.

\subsection{Objetivos}

Com base nos trabalhos citados neste artigo, procurou-se desenvolver um protótipo de um sistema de automação residencial, podendo assim, este sistema, ser ajustado às necessidades de uma residência ou para qualquer outro tipo de edificação que necessite de um sistema de automação. Em que esse sistema possibilite a utilização do padrão IEEE 802.15.4 (ZigBee) para comunicação entre os atuadores na automação residencial, como também o uso de softwares de gerenciamento de código aberto para a coleta de informações pertinentes a automação residencial e a alteração desses status. 


\title{
2 TRABALHOS RELACIONADOS
}

$\mathrm{Na}$ literatura estão presentes diversos trabalhos que tratam do monitoramento e automação residencial, os quais apresentam diferentes métodos. Todos eles buscam garantir maior conforto e segurança aos usuários, como apresentado por Dias e Pizzolato (2004), onde o sistema domótico é composto de uma rede de comunicação que permite a interconexão de uma série de dispositivos, equipamentos e outros sistemas, com o objetivo de obter informações sobre o ambiente residencial, objetivando prover maior comodidade e segurança em relação ao usuário.

Um projeto apresentado por Gomes (2011) estabelece a automação remota de um sistema de dispositivos que simula o acionamento de aparelhos eletro/eletrônicos de uma residência, trazendo mais uma facilidade e comodidade para o dia a dia utilizando a tecnologia IEEE 802.15.4 ZigBee.

\begin{abstract}
A partir da observação das necessidades do dia a dia de algumas pessoas com dificuldade de locomoção, com o intuito de proporcionar maior conforto e inclusão de projetos de automação na construção civil é proposto um sistema de acionamentos de dispositivos de iluminação e ventilação de ambientes de uma residência, utilizando a comunicação wireless para acionar tais dispositivos, fazendo uso da tecnologia ZigBee. A interface computacional interage com o módulo coordenador que envia as informações à placa XBee -Pro cuja finalidade é encaminhar as solicitações do sistema para o módulo remoto. $O$ módulo remoto também contém um móduloXBee-Pro, além de um micro controlador. (GOMES, 2011)
\end{abstract}

O projeto apresentado por Marchesan (2012) tem a proposta de utilizar a plataforma Arduino para possibilitar o monitoramento de diversos sensores e módulos que possibilitem a automação residencial. Esse projeto desenvolveu um sistema autônomo de gerenciamento de segurança residencial de baixo custo, confiável e eficiente que possa ser acessado através da Internet.

Segundo Nova (2009), A arquitetura de um Sistema de Automação Residencial é toda modular. Os módulos são subsistemas independentes que se comunicam através de uma rede de sensores e atuadores. Essa arquitetura facilita a distribuição dos módulos que compõem o sistema (HADDON, 1995) e (DELGADO, 2005). Essa divisão entre módulos facilita na montagem do esquema para a automação residencial.

Existem vários tipos de módulos, e eles são chamados de Clusters na especificação ZigBee (ZIGBEE, 2007). Um conjunto de clusters pode formar um perfil. Um exemplo de perfil, é o de Automação Residencial, que pode ser composto por clusters de lluminação, de HVAC (Heating Ventilation and Air Conditioning), de Sensoriamento, dentre outros módulos. Com a utilização de clusters pode-se dividir a automação em segmentos, por exemplo, iluminação, climatização, áudio e vídeo. Possibilitando um maior controle desses nodos na rede. Essa facilidade trazida pela arquitetura ZigBee 802.15.4 possibilita agilidade e gerenciamento do sistema de automação residencial.

Para a aplicação no sistema de automação residencial proposto pensa-se na comunicação dentre os nodos dos clusters, como deverá ser feita e que obstáculos são encontrados e colocados em questão para integração entre os nodos. Para a aplicação no sistema de automação residencial 
a tecnologia ZigBee apresenta ser a mais favorável por suas características apropriadas para essa finalidade.

O artigo apresentado por Araújo e Junior (2013) propõe montar um sistema aplicado a monitoramento que colete dados utilizando o protocolo de gerenciamento SNMP, com o fim de coletar dados para gerar dados estatísticos. A eficiência do protocolo SNMP possibilita um gerenciamento dos status dos nodos gerenciáveis de um sistema de automação residencial. Possibilitando a utilização de softwares de monitoramento para esse gerenciamento.

\section{METODOLOGIA}

Para validar as ideias de solução do problema identificado anteriormente foi desenvolvido um protótipo, envolvendo conceitos que pretendem a criação de um produto final que visa os resultados obtidos ao decorrer das pesquisas e testes realizados durante a produção deste artigo. Com finalidade de legitimar conhecimentos acadêmicos, para o fim de que se possa apresentar uma aplicação prática do uso da tecnologia ZigBee para a comunicação juntamente ao protocolo de gerenciamento SNMP para captura dos dados e o software ZABBIX para leitura e escrita desses dados.

\subsection{Materiais Utilizados}

Os dispositivos utilizados para o experimento realizado são: Uma placa Arduino versão Mega 2560, uma Ethernet Shield, uma Shield ZigBee, um roteador para servir como ponto de acesso, 2 módulos ZigBees Serie2, uma shield relê, um Arduino UNO e uma estação configurada com o software de recuperação de dados SNMP, ZABBIX, que é uma solução em código aberto.

\subsection{Arquitetura do Sistema}

Para os estudos desse artigo a arquitetura do sistema de automação residencial foi dividida entre três subsistemas, conforme ilustrado na Figura 1.

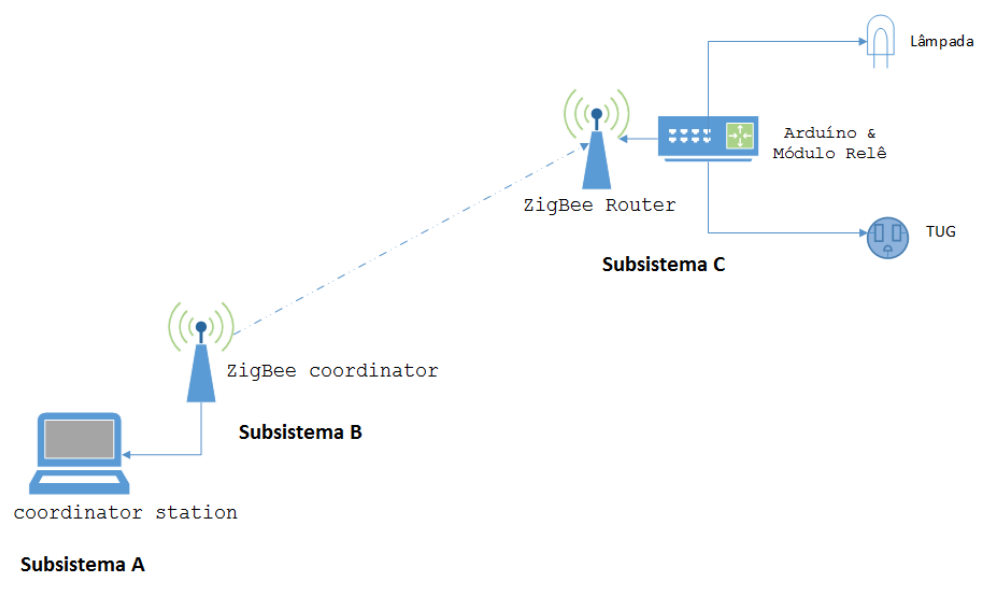

Figura 1 - Diagrama de interligação entre os subsistemas

O subsistema A apresenta a estação com o gerente SNMP para monitoramento dos dados capturados a partir do sistema de automação, e este por sua vez se comunica com o subsistema B por meio da comunicação Ethernet como demonstra a figura 2, que é composto de Arduino Mega 2560, uma Ethernet Shield, uma Shield ZigBee. 
O subsistema B é onde o protocolo SNMP trabalha por meio da biblioteca Agentuino, uma biblioteca SNMP para a plataforma Arduino, que coleta os dados dos pontos do subsistema C. Este por sua vez apresenta os pontos da automação a serem gerenciados. A comunicação entre o subsistema B e C é realizada por meio da comunicação entre os nodos ZigBees, como apresentado na Figura 1. O subsistema C é composto de um Arduino UNO, uma Shield ZigBee, e um módulo relê. Todo gerenciamento e acionamento de qualquer dispositivo acontecem no subsistema $A$.

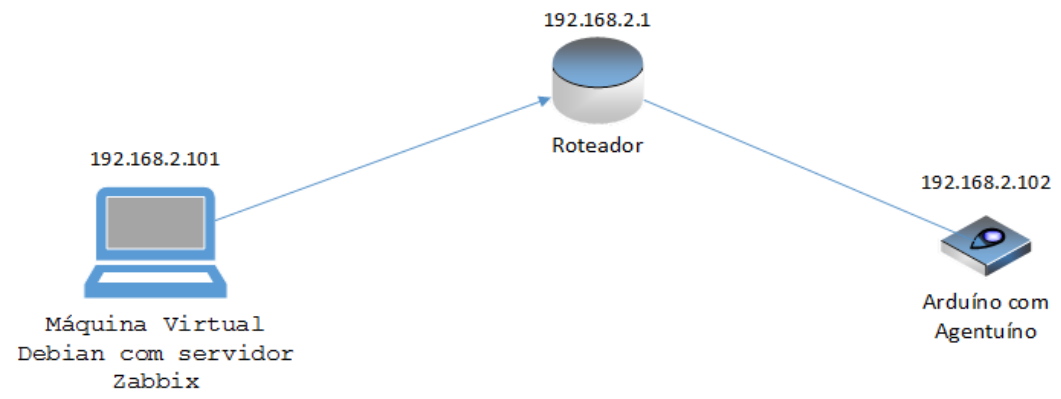

Figura 2 - Diagrama de interligação entre os subsistemas A e B

\subsection{Leitura e Escrita de Informações}

Como citado anteriormente o gerenciamento e acionamento dos dispositivos acontece no subsistema $A$, isso é feito por meio do software ZABBIX, que é um gerente SNMP, cada dis positivo do sistema de automação residencial, seja ele uma luminária ou uma tomada de uso geral, possui uma OID dentro do agente SNMP, que se encontra no subsistema B por meio da biblioteca Agentuino. Dessa forma o ZABBIX faz a leitura e a escrita dessas OIDs, utilizando os comandos GET, para consulta, ou SET, para alterar.

Com isso o ZABBIX pode identificar os status de uma luminária com o comando GET, ou alterar os status da luminária com o comando SET.

\section{VALIDAÇÃO DO PROTÓTIPO DESENVOLVIDO}

\subsection{Comunicação entre o Subsistema B e C}

Nos experimentos iniciais foram realizados a comunicação entre dois módulos ZigBee com o propósito de comunicação entre um ZigBee como coordenador localizado em estação gerente e o outro ZigBee como ponto final, localizado em uma base remota junto ao modulo relê que acionará os pontos no circuito elétrico residencial, um de iluminação e o outro de tomada de uso geral (TUG), o acionamento dos circuitos foram realizados por meio de uma placa com que possui dois relês, onde cada relê foi direcionado para o acionamento e interrupção da luminária ou da TUG. O módulo composto por dois relês foi ligado ao Arduino, onde o módulo utiliza a alimentação VCC de $5 v$ do Arduino e o GND, e o acionamento e interrupção de cada relê é feita utilizando os pinos digitais do Arduino.

O ZigBee foi utilizado para a comunicação entre o Arduino com a o módulo relê e uma estação remota para o acionamento e interrupção de cada ponto. Nesse experimento a estação gerente foi o computador, e os comandos eram passados através do serial monitor da IDE do Arduino. Utilizando a sintaxe de " $\mathrm{d}$ " para desligado e "I" para ligado. Os testes apresentados aqui foram realizados com sucesso. 


\subsection{Comunicação entre o Subsistema A e B}

Neste experimento foi aferida a comunicação entre o subsistema A e B, essa comunicação é realizada por meio do protocolo Ethernet, em que o subsistema A possui um IP (Internet Protocol) e o subsistema $B$ também possui um outro endereço IP, permitindo assim que o ZABBIX que situase no subsistema $A$ encontre por meio deste protocolo o subsistema $B$, e dessa forma possibilitando o uso do protocolo SNMP.

Para que a configuração de rede dos Subsistemas A e B sejam feitas corretamente é necessário definir alguns parâmetros em sua rotina, são estes: o endereço de rede IP, o endereço MAC (Media Access Control), a máscara de sub-rede e o Gateway padrão (ponto de conexão) que neste caso é o endereço do ponto de acesso. No ponto de acesso como ilustrado na Figura 2 foram reservados os IPs para o subsistema A como também para o subsistema $B$.

\subsection{Validação do Agente SNMP na Plataforma Arduino}

O monitoramento dos dispositivos controlados pelo Arduino foi realizado pelo Zabbix, como também foi utilizado o protocolo SNMP para coleta das informações para o monitoramento, como as informações foram coletadas do Arduino é necessário um agente SNMP, a plataforma Arduino possui uma biblioteca chamada "Agentuino" que tem essa função.

Com isso pode-se utilizar o ZABBIX (Software em código aberto para gerenciamento de rede) para monitorar e acionar os relês que controlam os circuitos elétricos. A biblioteca Agentuino depende de outras três bibliotecas: Streaming Library, Flash Library e MemoryFree Library, sendo esta última biblioteca responsável pela contagem de memória disponível no Arduino.

No código do Arduino pode-se associaruma OID (Object Identification) a uma variável, onde essa variável poderá ser uma identificação no código de um dispositivo monitorado e gerenciado no sistema de automação residencial. Dessa forma o ZABBIX irá monitorar essa OID, verificando ou alterando os seus status.

\subsection{Coleta e Alteração das Informações}

As informações são coletadas por meio do protocolo SNMP, o ZABBIX é quem verifica essas informações consultando as OIDs do agente SNMP no Arduino, tal rotina é composta por uma função que verifica e modifica o valor de uma variável, à medida que o usuário solicita no ZABBIX a mudança desse status, essa mudança no sistema de automação pode ser o ligamento ou desligamento de uma luminária, quando solicitada a mudança de status o ZABBIX executa um script que muda o valor de uma OID que no qual está atribuída a uma variável contida em um função do Arduino, que reconhece essa alteração e por meio de um condicional que liga ou desliga determinado circuito.

\section{DISCUSSÃO DOS RESULTADOS}

As práticas iniciais foram realizadas para validar por meio de tes tes a comunicação Ethernet entre o subsistema $A$ e $B$, visto que a falta de comunicação entre esses subsistemas impossibilita 0 acionamento e monitoramento do subsistema $\mathrm{C}$. 
Esse teste é importante, pois verifica se há comunicação entre os subsistemas $A$ e $B$, por meio desse teste que foi realizado utilizando o comando ping, a partir do terminal da estação pertencente ao subsistema A, que utiliza o sistema operacional Windows 7 de 64 bits, que pode ser verificado na figura 3, com isso obteve-se êxito no teste e a comunicação entre os subsistemas foi realizada, sendo assim validada o tes te de comunicação.

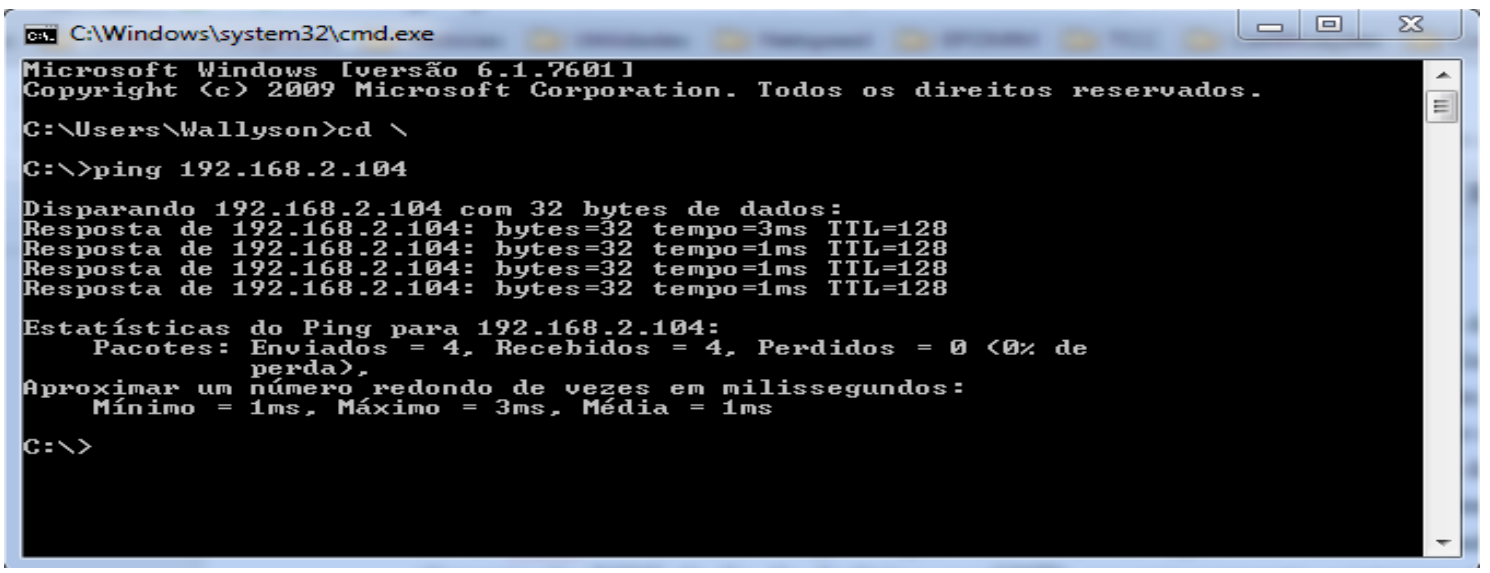

Figura 3 - Teste de comunicação entre os subsistemas A e B

Por meio desse teste pretende-se identificar a comunicação entre os módulos ZigBees, como também, o nível de sinal verificando a operacionalidade do subsistema $C$. Na sequência foi realizado o teste de comunicação entre o subsistema $B$ e $C$ que acontece por meio do módulo ZigBee, utilizando o software X-CTU, que pode ser observado na figura 4.

Com esse teste verificou-se a comunicação entre os subsistemas $B$ e $C$ que utilizam os módulos ZigBee, como também, o nível de sinal, que se mostrou satisfatório, permitindo assim, comunicação entre módulos.

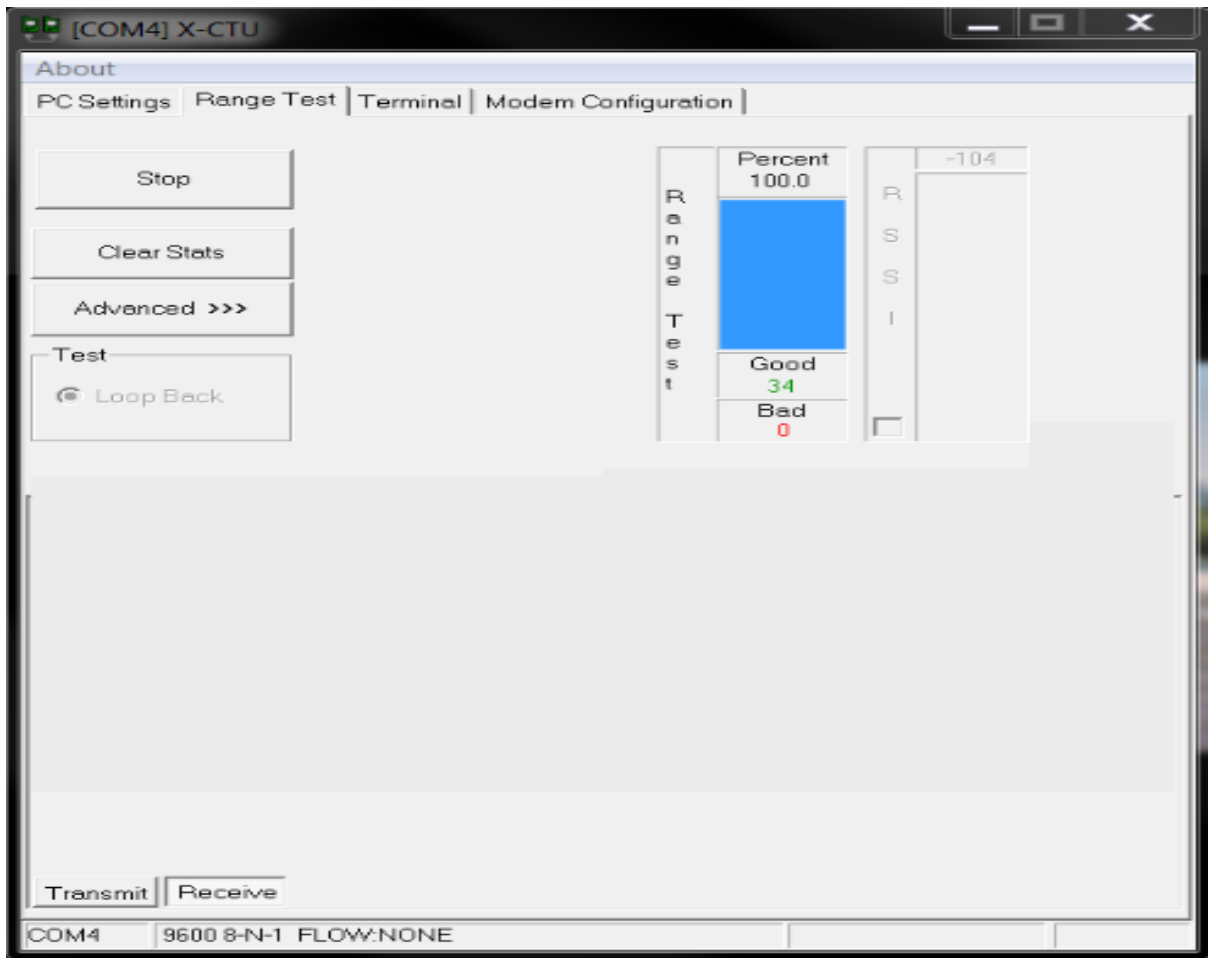

Figura 4 - Nível de sinal entre os subsistemas B e C 
O teste seguinte consiste na coleta de informações utilizando o agente SNMP, para isto foi utilizada a ferramenta: Agente NET-SNMP, com esta ferramenta é possível fazer a leitura e escrita dos dados no formato SNMP dos agentes.

Para esse teste foi utilizado o NET-SNMP que por meio de comandos de textos utilizando o terminal de comandos que permite a visualização e modificação das informações contidas na OID, a figura 5 retorna o valor da OID em teste.

C: $:$ Windows $\backslash$ system $32 \backslash \mathrm{cm}$.exe

\begin{tabular}{l|l|l}
\hline & 回 & $\times 3$ \\
\hline
\end{tabular}

C: $>>$ snmpget $-\mathrm{u} 1$-r 1 -c public 192.168.2.104 1.3.6.1.4.1.36582.3.1.0

SNMPU2-SMI : : enterprises $.36582 .3 .1 .0=$ INTEGER: 1

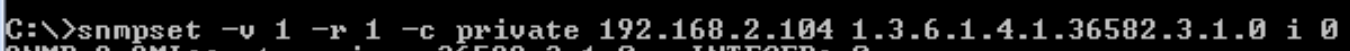
SNMPU2-SMI : : enterprises . $36582.3 .1 .0=$ INTEGER: 0

$\mathrm{C}: \backslash>$ snmpget $-\mathrm{u} 1-\mathrm{r} 1-\mathrm{c}$ public 192.168.2.104 1.3.6.1.4.1.36582.3.1.0

SNMPU2-SMI : : enterprises . $36582.3 .1 .0=$ INTEGER :

$C: \backslash>$

Figura 5 - Resultados de consulta e escrita a OID

Com a alteração desses dados da OID, que são realizadas por meio de scripts noZABBIX pelo usuário situado no subsistema $A$, o código do Arduino contido no subsistema $B$ capta essa alteração de valor e aciona o módulo relê. Essa alteração de valores por meio do script pode ser observada conforme a Figura 6.

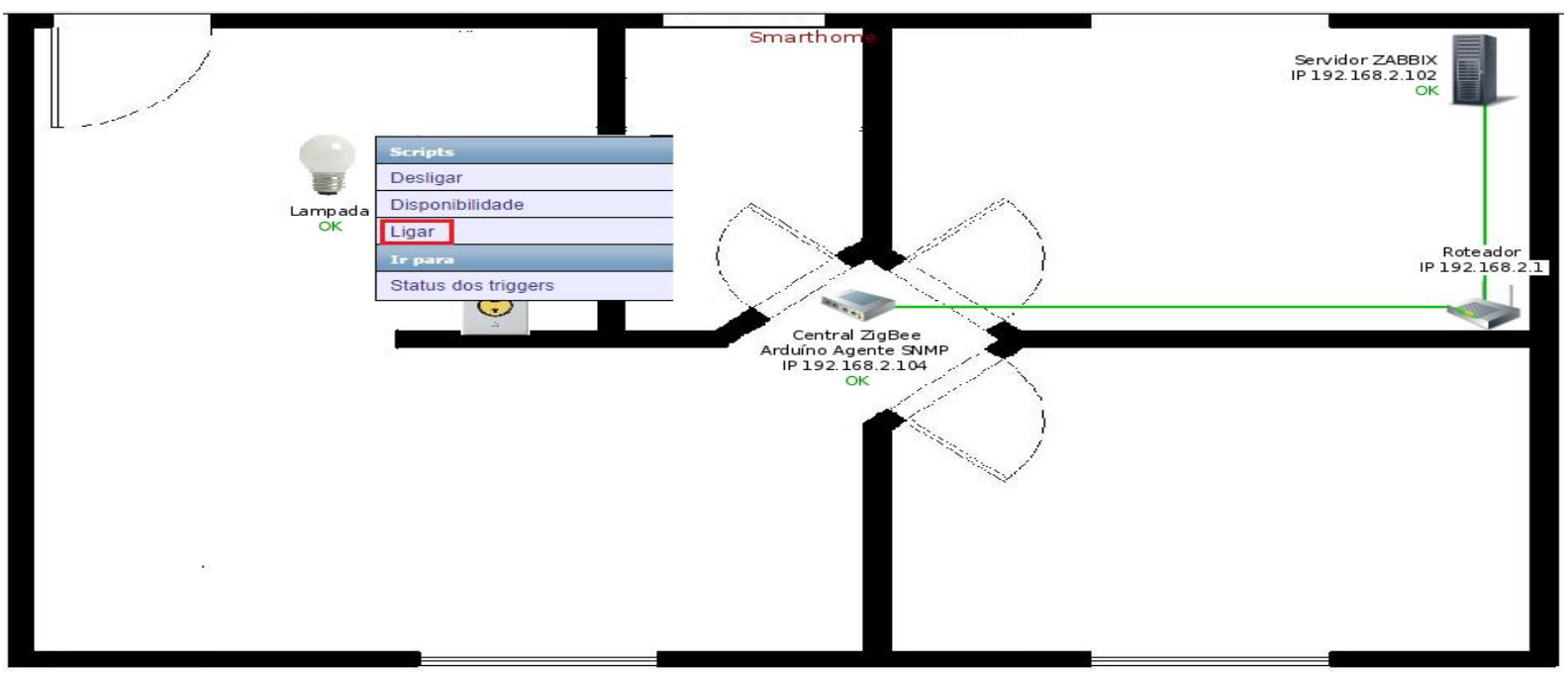

Figura 6 - Script “Ligar” no ZABBIX para alterar o valor da OID 
A partir de scripts executados no ZABBIX, o Arduino possui uma função que detecta a alteração do valor e muda o status do relê de acordo com o valor atribuído, pode-se analisar essa etapa a partir do pseudocódigo ilustrado na Figura 7.

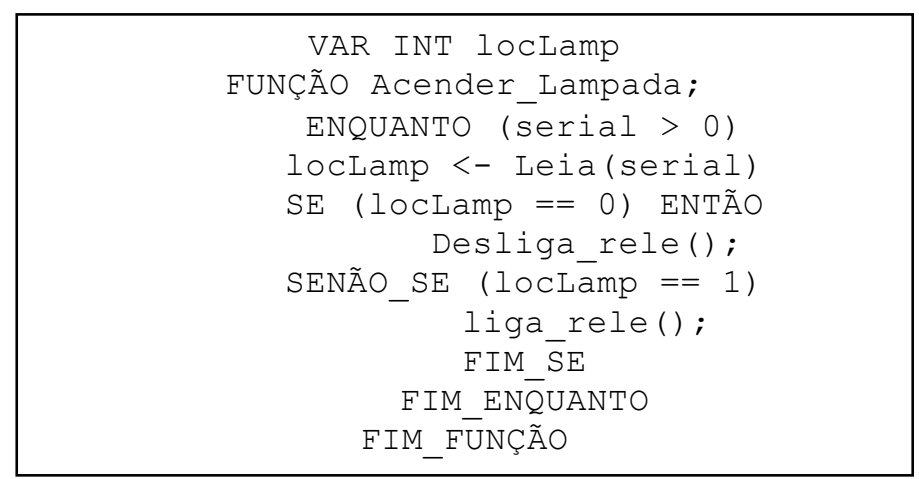

Figura 7 - Pseudocódigo que aciona o relê após mudança da OID

\section{CONCLUSÃO}

O sistema para automação residencial apresentado nesse artigo, que utiliza as tecnologias ZigBee, SNMP e ZABBIX comportou-se de forma satisfatória, obtendo êxito nos testes no qual foi submetido, nos testes realizados com o ZABBIX, o mesmo mostrou-se uma ferramenta aceitável. Apesar de ter sido idealizada para ser utilizada como uma ferramenta para administração de redes de computadores, a partir do ZABBIX foi possível obter o tempo de monitoramento, tempo de resposta e o tempo que o sistema permaneceu funcionando em um período de tempo. Essa forma de automação residencial atendeu os requisitos dispostos nesse documento, por utilizar o padrão ZigBee, sendo um meio de comunicação sem fio, dessa forma, facilitando na instalação e aumento no alcance do sistema, podendo assim atingir áreas externas de uma residência sem muita complexidade. Outro fato de suma importância é a utilização do gerente ZABBIX juntamente ao protocolo SNMP, permitindo o gerenciamento dos dispositivos que fazem parte do sistema de automação residencial. Somando essas ferramentas pode-se obter uma aplicação personalizável, aberta e de baixo custo quando comparada com outras tecnologias existentes no mercado. Apesar de um fim acadêmico, o protótipo apresentado possui possibilidade de utilização e melhoramento para fins comerciais.

A partir dos resultados obtidos o sistema permite ser adaptado a qualquer residência, ou outro tipo de edificação, e tem como característica a expansão da rede em relação ao número de dispositivos gerenciados como também a forma de gerenciamento devido a gama de recursos permitidos pelo software ZABBIX.

\section{REFERÊNCIAS BIBLIOGRÁFICAS}

1. BORGES, L. P.; DORES, R. D. Automação Predial sem Fio Utilizando BACNET/ZIGBEE com Foco em Economia de Energia, (2010), Brasília, DF, Brasil.

2. DIAS, C. L.; PIZZOLATO, N. D. Domótica Aplicabilidade e Sistemas de Automação Residencial, (2004), Campos dos Goytacazes. Rio de Janeiro, Brasil.

3. GOMES, H. G. Automação Residencial usando tecnologia IEEE 802.15.4, (2011), Brasília, DF, Brasil. 
4. Marchesan, M. Sistema de Monitoramento Residencial utilizando a Plataforma Arduíno, (2012), Santa Maria, Rio Grande do Sul, Brasil: UFSM.

5. ARAÚJO, A. C.; JUNIOR, I. F. Protocolo de Transmissão de Dados SNMP em Redes de Sensoriamento, (2013) Natal, Rio Grande do Norte, Brasil.

6. NOVA, F. S. Redes de Sensores em Malha sem Fio: Um Estudo de Caso de Automação Residencial usando a Tecnologia ZIGBEE/IEEE 802.15.4, (2009), Belém, Pará, Brasil: UFPA. 